\title{
Les Quand-P valencielles et les structures corrélatives
}

\author{
Frédérique $\mathrm{Saez}$ \\ CLLE-ERSS \\ Université de Toulouse II Le Mirail et CNRS \\ 5, allée Antonio Machado \\ $\mathrm{F}-31058$ TOULOUSE CEDEX 9 \\ saez@univ-tlse2.fr
}

\section{Introduction}

Les propositions introduites par quand, sont traditionnellement considérées comme des propositions circonstancielles étant dans la dépendance d'un prédicat verbal. Or des travaux linguistiques plus ou moins récents (JeanJean, Borillo, Benzitoun...) montrent qu'il existe, en fait, de nombreux modes d'intégration de Quand-P dans un énoncé.

Nous restreindrons notre cadre d'étude aux Quand- $P$ analysées comme appartenant à la valence, sujet ou objet, d'un verbe ${ }^{1}$, ainsi que dans les propositions suivantes :

(1) Ça me surprend toujours quand des êtres humains se comportent ainsi (exemple (630) donné par C. Benzitoun (2007 : 211))

(2) J'aime quand tu souris

(3) Elle m'a parlé de quand vous étiez petits (M. Aymé, cité dans Le Petit Robert)

En position argumentale, la Quand-P est très fortement nominalisée, d'où sa proportionnalité avec une proforme ça/cela/le, et entre dans le paradigme suivant (nous verrons que l'analyse de Quand-P en position de sujet pose un certain nombre de problèmes, nous ne donnons donc ci-après que le paradigme de la valence objet) :

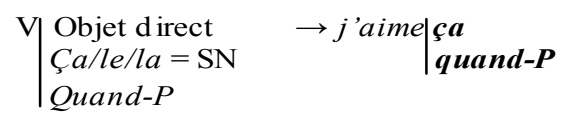

Dans cette étude nous comparerons cette position argumentale - directe ou prépositionnelle - avec d'autres types de Quand-P, que nous reconnaitrons comme des Quand-P corrélatives, qui sont déterminées (notamment par Benzitoun (2007)) comme étant concurrentielles des précédentes. Il s'agira donc pour nous de définir les deux types de structures, valencielle et corrélative, et de les comparer afin de comprendre la raison de leur présence simultanée en langue, puisque nous ne croyons pas en la « synonymie », tant sémantique que syntaxique.

Notre cadre théorique ne sera pas contraint à celui de l'Approche Pronominale, quoique nous reprendrons la terminologie de cette approche, car nous ne pensons pas la syntaxe comme indépendante des théories pragmatiques et sémantiques, aussi nous ne définirons qu'au fur et à mesure les notions auxquelles nous aurons recours et notre étude se fera sous deux angles corrélés: syntaxique et sémantico-discursif. Concernant le corpus, les exemples sont essentiellement extraits de corpus écrits (Frantext, Le Monde), les exemples de français parlé étant empruntés à Benzitoun (2007), mais à l'inverse de son choix méthodologique, nous avons écarté de notre analyse les Quand-P interrogatives, pensant que ces dernières doivent faire l'objet d'une étude particulière.

Avant d'aborder l'étude proprement dite des Quand-P valencielles et corrélatives, il nous parait nécessaire de définir ce que nous entendons par «structure corrélative ». 


\section{Qu'est-ce qu'une « structure corrélative »?}

Nous définirons ce schème sur le modèle de S. Allaire (1982: 427) comme un mode de « codétermination syntaxique bilatérale », c'est-à-dire défini à la fois par l'incomplétude syntaxique et «l'équipotence » des deux verbes inclus dans les deux parties constituant l'énonciation unaire.

Ce tour syntaxique a été décrit en diachronie par Haudry (1973) qui le situait à un niveau intermédiaire entre la parataxe et l'hypotaxe: pour lui, les deux parties de l'énonciation sont interdépendantes, et aucune n'est à considérer comme subordonnée. La structure peut s'exprimer segmentalement par des signifiants différenciés, ou identiques, et c'est le cas du latin, langue pour laquelle la forme binaire de base de la corrélation est le diptyque normal, où le relatif précède la proposition introduite par le corrélatif anaphorique (le diptyque inverse étant considéré par Haudry (1973) comme un effet de style), ce que l'on peut rendre schématiquement de la façon suivante :

[pronom relatif + pronom de reprise anaphorique $]=$ i.-e. $\left[{ }^{*} k^{w} o-\ldots{ }^{*}\right.$ to $]$, lat. qui ...is.

Ce qui donne par exemple :

(4) Lat. "Quos ferro trucidari oportebat, eos nondum voce vulnero » (Cicéron, [cité par Haudry]) Fr. : Des gens qu'il aurait fallu massacrer par le fer, (ceux-là) je ne les blesse pas

S. Allaire dans sa thèse (1982) confirme le caractère interpropositionnel du schème en posant le principe d'une double incomplétude sous-jacente et condition à l'unicité dialectique de la structure. Cette double incomplétude est marquée par la présence de termes corrélatifs qui entretiennent entre eux un rapport de dépendance interne (basée sur une incomplétude syntaxique (une $Q u$-forme introductive) et sur une incomplétude énonciative (une anaphore), formant les deux parties corrélatives d'un tout, signalant la complémentarité des propositions introduites. En termes discursifs et pour reprendre la terminologie de Charolles (1997), la $Q u$-forme induit une relation d'indexation, et l'anaphore une relation de connexion avec réinterprétation, réanalyse, de la $\mathrm{P} 1$, relations pour lesquelles A. Le Draoulec et $\mathrm{M}$. Bras précisent $(2005: 2)$ :

\footnotetext{
«La connexion [r] correspond à un mode de relation « vers l'arrière », au sens où un lien se crée avec ce qui précède [relation de type anaphorique]. L'indexation [r'], en revanche, désigne un processus qui s'établit «vers l'avant» [relation de type cataphorique] : l'expression indexante [...] constitue un critère d'interprétation pour la ou les propositions qui la suivent [...].»
}

La seconde incomplétude de la structure se situe à un niveau interne aux unités partielles ouvertes par les termes corrélatifs, donc au niveau essentiellement des verbes des deux prédications unies, qui sont donc articulés de telle sorte que l'ordination soit réciproque, et non pas hiérarchique.

Les P1 et P2 d'une structure corrélative sont donc dans une interdépendance syntactico-sémanticoréférentielle, puisque, comme le dit S. Allaire (1982: 122), «la réalisation anaphorique souligne la complémentarité des deux propositions : alors que le second complète séquentiellement le premier, celuici est repris comme complément du second »; l'anaphore est donc un indice fort de l'incomplétude entre les deux énoncés, et c'est de réinterprétation dont il s'agit au sein d'une structure corrélative : P1 ne se comprend qu'après l'énonciation de $\mathrm{P} 2$, qui ne s'interprète qu'à partir du «pôle de référence $»^{2}$ que constitue P1.

Ce que l'on peut représenter schématiquement de la façon suivante : 


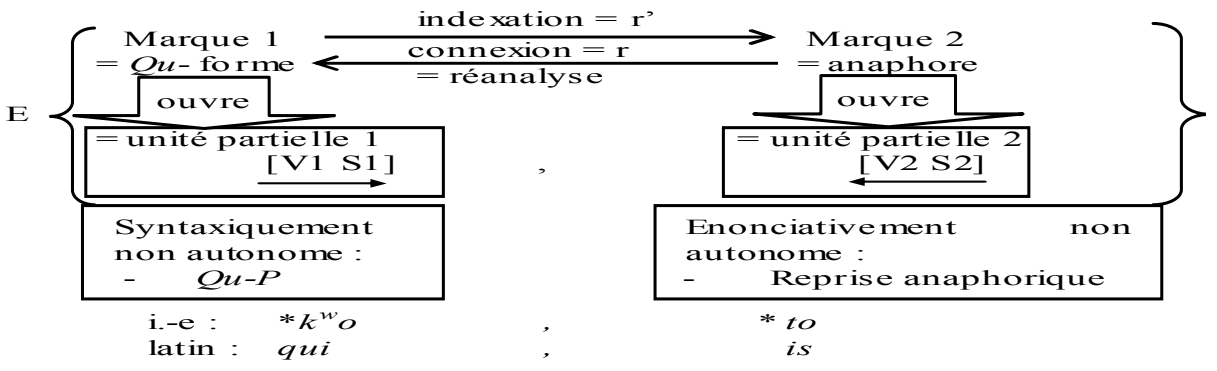

Figure 1

Pour notre analyse nous retiendrons les propriétés suivantes :

1. Les marques corrélatives doivent avoir une fonction syntaxique dans les propositions qu'elles introduisent (pour la $Q u-P$ ), ou dans lesquelles elles sont incluses (pour le pronom anaphorique) ;

2. Il faut qu'il y ait concomitance de deux indices d'incomplétude : un indice segmental ( $Q u$ - forme + pronom anaphorique) et un indice syntaxique (interdépendance des V1 et V2);

3. Les marques corrélatives doivent avoir un rôle de désignation de deux unités partielles (rôle assumé par la fonction syntaxique démarcative de la $Q u-P$ et par la fonction énonciative anaphorique du pronom) et fonctionnel (les unir au sein d'une énonciation unaire).

\section{Quand-P en position de valence sujet ou structure corrélative en [Quand...cela/ça] ou [cela/ça...Quand-P] ?}

C. Benzitoun (2007) avance l'idée d'une Quand-P en position sujet et interprète de cette façon les propositions (5) et (6) suivantes :

(5) Ça me surprend toujours quand des êtres humains se comportent ainsi (exemple (630) donné par C. Benzitoun (2007: 211))

(6) L1 non ce qu'il y a c'est que non quand on joue par exemple au bord de l'Atlantique + quand la mer se retire ça laisse un sable assez dur des fois + bien lisse et dur + (exemple (632) donné par C. Benzitoun (2007: 212))

Pour justifier de sa position, il cite Muller (1996a : 217) qui précise sur ce type de Quand-P qu'elles viennent, par le biais de la reprise pronominale «se nicher dans une position fonctionnelle à laquelle [elles] n'[ont] en principe pas droit », en l'occurrence la position argumentale de sujet.

C. Benzitoun (2007) va plus loin en postulant la possibilité de trouver en langue la construction inventée ci-dessous :

(7) ? "quand arrêter les hostilités des deux côtés de la frontière (constitue/représente) un sujet délicat lourd de conséquence. » (C. Benzitoun ; ex : (636) (2007: 213)).

Mais il précise, et nous confirmons le même échec, n'avoir pas trouvé en corpus (ni oraux, ni écrits) d'attestation de ce type d'emploi de Quand-P.

De plus le parallélisme établi par Benzitoun entre les exemples (5) et (6) et l'exemple (7) nous parait peu justifié, et s'il l'était nous pourrions alors remplacer pour les propositions (5) et (6), le pronom ça par la Quand-P (comme nous verrons qu'il est possible de faire pour les Quand-P en position de valence directe). Or cette opération rend les énoncés (5) et (6) agrammaticaux, comme le montrent les manipulations (5a) et (6a) ci-dessous : 
(5a) ? $?^{3}$ quand des êtres humains se comportent ainsi me surprend toujours

(6a) * quand la mer se retire laisse un sable assez dur des fois

De surcroit pour son exemple (7) la substitution possible de quand par lorsque - ou encore à quel moment - pousse à une interprétation interrogative pour ce quand, ce qui invaliderait nécessairement son hypothèse :

(7a) à quel moment/lorsqu'arrêter les hostilités des deux côtés de la frontière constitue/représente un sujet lourd de conséquences?

Substitution impossible pour les autres propositions :

$(5 b)$ * à quel moment/lorsque des êtres humains se comportent ainsi me surprend toujours ?

(6b) * à quel moment/lorsque la mer se retire ça laisse un sable assez dur des fois ?

Si son interprétation était justifiée, nous serions alors en présence d'une Quand-P thématisée, rejetée à gauche et, dans ce cas, il ne nous serait pas possible, comme le signifie B. Combettes (1996: 92), d'insérer l'adverbe modalisateur certes entre la principale et la subordonnée thématisée ${ }^{4}$, or pour nos propositions (5) et (6) il est possible d'intégrer certes :

(5c) quand des êtres humains se comportent ainsi, certes ça me surprend toujours

(6c) quand la mer se retire, certes ça laisse un sable assez dur des fois

C'est donc que la proposition introduite par quand n'est pas une temporelle thématisée, rejetée à gauche et dont la reprise pronominale serait la marque du déplacement, mais qu'elle est topicalisée. Nous nous appuyons ici sur le modèle de Combettes (1996), (1998) et opposons les notions de thème et topique en ce que le thème correspond au constituant le moins informatif sur l'échelle du dynamisme communicatif, et le topique au «point de départ de la communication » (Combettes (1998: 138)), c'est-à-dire à ce à partir de quoi peut s'opérer un « commentaire », une prédication.

C'est sur ce principe de la topicalisation de la proposition conjonctive que nous pouvons postuler une interprétation corrélative pour ces structures.

Bien que l'on trouve de nombreux exemples de diptyque inverse, nous analyserons essentiellement ici, pour des raisons de place, le diptyque normal en [quand...cela/ça] dont les propositions (8), (9) et (10) suivantes :

(8) Quand j'ai bu, ça se voit; quand j'ai soif, ça se voit pas (exemple (646) donné par C. Benzitoun (2007: 215))

(9) Quand un connard se fait étendre, ça fait jamais de vagues. C'est bon pour moi. Et pour toi aussi. Si je te plombe, tes copains prendront ma doublure. (Frantexte : J-Cl. Izzo, Total Khéops (1995 : 213-214))

(10) Je ne déteste pas que dans la controverse ce gavé de science se montre si fort attaché à son opinion qu'il cesse enfin d'être raisonnable. Bref, quand il règne, ça baigne. Mais raide, il n'est pas sec: il tient à sa fratrie, qui n'est pas sans pouvoir sur lui, surtout Yvonne. (Frantexte: H. Bazin, L'école des pères (1991:224))

pour lesquelles l'insertion de l'adverbe certes ne pose pas de problèmes, et que l'on ne saurait donc analyser comme des temporelles thématisées :

(8a) quand j'ai bu, certes ça se voit ; quand j'ai soif, certes ça se voit pas

(9a) quand un connard se fait étendre, certes ça fait jamais de vagues

(10a) quand il règne, certes ça baigne.

En diptyque normal, a priori, nos propositions semblent correspondre aux critères énoncés précédemment pour une structure corrélative, à savoir : 
1. Les termes corrélatifs doivent avoir une fonction dans la proposition qu'ils introduisent (ce que l'on peut mettre en évidence par la commutation de quand par au moment où, la fonction de cela/ça sujet ne posant pas de problèmes) :

(6d) quand/au moment où la mer se retire, ça laisse un sable assez dur

(8b) quand/au moment où $\mathrm{j}$ 'ai bu, ça se voit; quand/au moment où $\mathrm{j}$ 'ai soif, ça se voit pas

(9b) quand/au moment où un connard se fait étendre, ça fait jamais de vagues

$(10 \mathrm{~b})$ quand/au moment où il règne, ça baigne.

Nos propositions vérifient donc la première des propriétés, voyons ce qu'il en est des autres critères à savoir: 2. la concomitance de deux indices d'incomplétude ; et 3. le rôle à la fois démarcatif et fonctionnel des marques corrélatives.

Concernant la double incomplétude - segmentale et syntactico-énonciative - de la structure corrélative, on constate que toutes nos propositions sont constituées segmentalement d'un morphème quand introducteur démarcatif et fonctionnel, en ce qu'il désigne la dépendance - syntaxique et discursive - de la proposition qu'il introduit par rapport une autre proposition. Ce point est vérifiable par l'extraction de Quand-P dans une construction clivée :

(6e) c'est quand la mer se retire que ça laisse un sable assez dur

(8c) c'est quand j'ai bu que ça se voit ; c'est quand j'ai soif que ça se voit pas

(9c) c'est quand un connard se fait étendre que ça fait jamais de vagues

$(10 \mathrm{c})$ c'est quand il règne que ça baigne.

Les secondes parties de nos structures sont toutes introduites par les pronoms ça et cela, qui reprennent anaphoriquement la situation donnée dans les Quand-P antéposées, indiquant par cette voie l'incomplétude sémantique des propositions qu'ils introduisent. Nous parlons ici non d'anaphore totale, mais d'anaphore que nous pourrions dire «associative», effectivement ce n'est pas la « temporalité » de la Quand-P qui est reprise anaphoriquement, mais la situation donnée, ce que l'on pourrait paraphraser de la façon suivante :

(6f) quand / le fait que la mer se retire, (ça) laisse un sable assez dur $\rightarrow$ quand la mer ce retire, ça/ce fait laisse un sable assez dur

(8d) quand / le fait que j'ai bu (ça) se voit; quand / le fait que j'ai soif ça se voit pas $\rightarrow$ quand j'ai bu ça/ce fait se voit ; quand j'ai soif ça/ce fait ne se voit pas

(9d) quand / le fait que un connard se fait étendre, ça fait jamais de vagues $\rightarrow$ quand un connard se fait étendre ça/ce fait ne fait jamais de vagues

(10d) quand / le fait que il règne, ça baigne $\rightarrow$ quand il règne ça/ce fait baigne

Cette interprétation situationnelle de Quand-P est soutenue par la difficile acceptabilité de la pronominalisation de Quand-P par ce moment-là :

$(6 \mathrm{~g})$ ? ce moment-là ça laisse un sable assez dur des fois

(8e) ? ce moment-là ça se voit ; ce moment-là ça se voit pas

(9e) ? ce moment-là ça fait jamais de vagues

(10e) ? ce moment-là, ça baigne

Le pronom anaphorique ne semble donc pas pointer un «moment » mais un état ou une situation donnée dans le cadre temporel ouvert par Quand, ce qui nous amène naturellement à nous demander si quand a toujours une valeur temporelle dans ces propositions, question soutenue par la commutation possible du morphème avec le fait que. Au niveau segmental donc nos propositions ne paraissent pas poser de problèmes, en revanche les choses sont plus complexes en ce qui concerne l'incomplétude des V1 et V2.

Tous nos procès sont dans un rapport de synchronisation qui peut être mis en évidence par le remplacement de quand par tant que - manipulation effectuée sur le modèle d'A. Borillo (1988) :

(6h) quand/tant que la mer se retire ça laisse un sable assez dur 
(8f) quand/tant que j'ai bu, ça se voit ; quand/tant que j'ai soif, ça se voit pas

(9f) quand/tant qu'un connard se fait étendre, ça fait jamais de vagues

(10f) quand/tant qu'il règne, ça baigne.

Cette contrainte à la synchronisation dans l'articulation entre V1 et V2 s'accompagne nécessairement d'une coréférence temporelle obligatoire, et l'on ne peut faire varier le temps de V1 ou V2, sans faire varier le temps du V2 ou V1 correspondant:

(5i) ? quand la mer se retirait/retirera, ça laisse un sable assez dur /? quand la mer se retire, ça laissait/laissera un sable assez dur

$(8 \mathrm{~g})$ ? quand je buvais/boirai, ça se voit; quand j'avais/aurai soif, ça se voit pas / ? quand je bois, ça se voyait/verra ; quand $j$ 'ai soif, ça se voyait/verra pas

$(9 \mathrm{~g})$ ? quand un connard se faisait/fera étendre, ça fait jamais de vagues / ? quand un connard se fait étendre, ça faisait/fera jamais de vagues

$(10 \mathrm{~g})$ ? quand il régnait/règnera, ça baigne / ? quand il règne, ça baignait/baignera.

Si cette contrainte de coréférence temporelle et de synchronisation n'est pas propre aux structures corrélatives, elle participe tout de même à désigner l'interdépendance des V1 et V2, indiquant, de fait, que les deux procès sont intimement liés au plan énonciatif.

Nos deux unités partielles s'articulent temporellement dans une sorte de parallélisme dont quand marque le point de départ, dans la mesure où il construit le pôle de référence dans lequel vient s'ancrer la temporalité de V2. Quand indique donc un point de contact temporel, moment à la fois précis (point de départ du parallélisme) et indéterminé (dont la temporalité n'est pas datée dans le temps) qui va définir la temporalité de V2 en le construisant dans un rapport de synchronisation aspectuo-temporelle qui provoque alors l'incomplétude de ce dernier.

Ce parallélisme temporel est soutenu par la topicalisation de la Quand-P qui induit une coupure informative par rapport à ce qui précède, coupure dans laquelle la structure corrélative s'insère comme une unité dialectique indépendante, construite sur le couplage de deux procès réciproquement ordonnés.

Nous pouvons donc établir le schéma suivant :

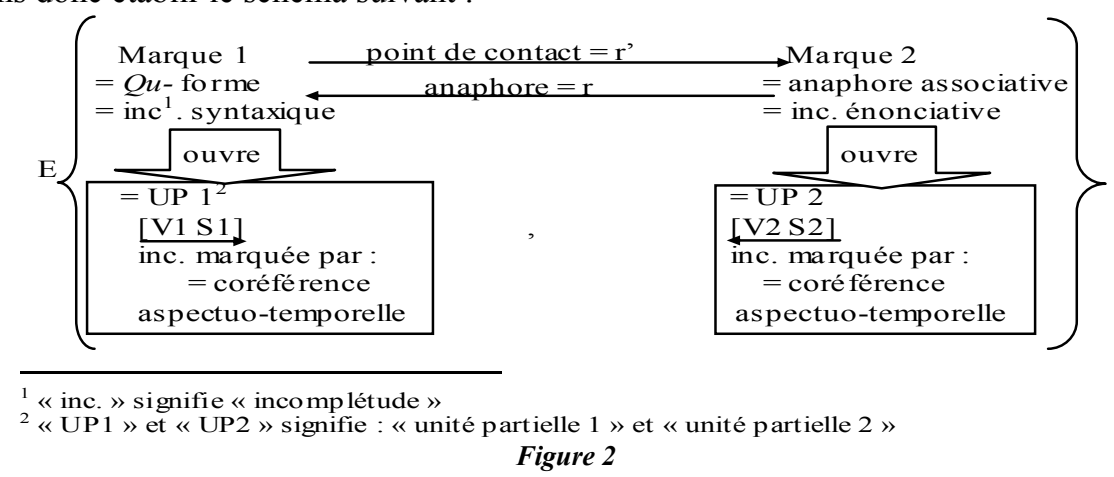

Le connecteur Quand assure, dans cette structure, le parallélisme organisationnel (segmentation) et sémantique (coupure avec ce qui précède et instruction d'un point de contact entre les V1 et V2).

\section{Quand-P en position de valence directe :}

Dans une telle position des contraintes fortes semblent peser à la fois sur la Quand-P et sur le prédicat. Effectivement on ne les trouve que dans la valence de verbes psychologiques ou de sentiment de type aimer, détester, préférer, adorer... : 
(11) J'aime aussi beaucoup quand il parle d'histoire naturelle. (A. Gide, cité dans le Petit Robert)

(12) Je déteste quand on m'en veut (Frantexte: S. De Beauvoir, Les mandarins, (1954:166))

(13) Moi je préfère quand ils crèvent tous (Frantexte : R. Queneau, Zazie dans le métro, (1959:224-225))

Outre la construction argumentale directe, C. Benzitoun (2007) signale pour ces verbes trois « groupes de constructions $^{5} \gg$ possibles dont un avec reprise pronominale de la Quand-P dans la valence objet du verbe :

(14) Quand je vois encore des jeunes femmes + autour du marché des Capucins chercher dans les poubelles + pour ramasser un morceau de boudin + que le le charcutier au lieu de leur donner a mis sur la poubelle + j’apprécie j’apprécie pas ça (exemple (638) donné par C. Benzitoun (2007 : 213)

(15) Pour moi, un magistrat responsable, c'est quelqu'un qui, quand il est amené à commettre une faute dans l'exercice de ses fonctions, le reconnaît et en répond (journal Le Monde, décembre 1999)

(16) Nous n'avons pas la formule magique pour les battre, mais je peux vous assurer que, quand vous perdez deux fois contre une même équipe, vous n'aimez pas cela du tout (journal Le Monde, décembre 1999)

Le parallélisme entre ces deux types de construction proposé par C. Benzitoun n'est pas sans fondement, et le premier constat que l'on peut faire pour appuyer cette analogie est que la position de la Quand-P n'est pas contrainte, ni pour l'une ni pour l'autre des propositions, à la post- ou antéposition (pour des raisons de place nous n'opérerons les tests que sur les propositions (11) et (14), mais ils sont aussi valables pour les propositions (12), (13), (15) et (16)) :

(11a) quand il parle d'histoire naturelle j'aime aussi beaucoup

(14a) + j'apprécie j'apprécie pas ça quand je vois des jeunes femmes + autour du marché des Capucins chercher dans les poubelles + pour ramasser un morceau de boudin + que le le charcutier au lieu de leur donner a mis sur la poubelle

De plus la reprise pronominale de la Quand-P pour (11) n'entraine pas l'agrammaticalité de l'énoncé :

(11b) quand il parle d'histoire naturelle, j'aime aussi beaucoup ça / j'aime aussi beaucoup ça, quand il parle d'histoire naturelle

De même que la construction « directe » de Quand-P pour (14) :

(14b) j'apprécie pas quand je vois des jeunes femmes + autour du marché des Capucins chercher dans les poubelles + pour ramasser un morceau de boudin + que le le charcutier au lieu de leur donner a mis sur la poubelle

On remarque également la même contrainte de coréférence temporelle entre les V1 et V2, pour l'une et l'autre des constructions :

(11c) ? j'aimais/j' aimerai aussi beaucoup quand il parle d'histoire naturelle / ? j'aime aussi beaucoup quand il parlera/parlait d'histoire naturelle

(14c) ? quand je voyais/verrai des jeunes femmes + autour du marché des Capucins chercher dans les poubelles + pour ramasser un morceau de boudin + que le le charcutier au lieu de leur donner a mis sur la poubelle + j'apprécie j'apprécie pas ça / ? quand je vois des jeunes femmes + autour du marché des Capucins chercher dans les poubelles + pour ramasser un morceau de boudin + que le le charcutier au lieu de leur donner a mis sur la poubelle + j'apprécierai/appréciais pas ça

La relation temporelle articulant les deux procès parait être de l'ordre de la synchronisation, et l'on peut faire commuter sur l'axe paradigmatique quand par tant que (les propositions deviennent alors plus acceptables lorsque la Quand-P est antéposée, c'est pourquoi nous donnons les deux manipulations pour (11)) :

(11d) je déteste, quand/tant qu'on m'en veut $\rightarrow$ quand/tant qu'on m'en veut, je déteste 
(14d) quand/tant que je vois des jeunes femmes + autour du marché des Capucins chercher dans les poubelles + pour ramasser un morceau de boudin + que le le charcutier au lieu de leur donner a mis sur la poubelle + j'apprécie j'apprécie pas ça

Avec ce type de verbes les locuteurs ont donc le choix entre une construction de valence directe, ou bien une structure avec reprise pronominale. Cependant peut-on considérer ces deux constructions comme syntaxiquement équivalentes? Et si non, la structure corrélative serait-elle dérivée de la structure directe ? La réponse à ces questions nous est offerte par l'adverbe certes que l'on peut insérer en (14), mais pas pour la construction valencielle directe :

(14e) quand je vois des jeunes femmes + autour du marché des Capucins chercher dans les poubelles + pour ramasser un morceau de boudin + que le le charcutier au lieu de leur donner a mis sur la poubelle + certes j'apprécie j'apprécie pas ça

Mais :

(11e) ? j'aime aussi beaucoup certes quand il parle d'histoire naturelle

Nous reconnaitrons donc, en (14), (15) et (16), une structure corrélative dans la mesure où l'on se trouve en présence d'un schème équivalent à ce que nous avions précédemment pour la construction [Quand...ça/cela] et qu'elles correspondent aux critères définis pour une telle structure, à savoir la concomitance de deux indices d'incomplétudes: 1. La présence des termes corrélatifs quand et ça assurant la ligature de l'énonciation et ayant une fonction au sein des propositions qu'ils introduisent ; et 2. L'incomplétude des V1 et V2 induite par la topicalisation de la Quand-P.

D'un point de vue sémantique les propositions (11), (12), (13) et (14), (15), (16) se distinguent. Effectivement, en (11), (12) et (13) l'interprétation d'un quand temporel ne pose pas de problèmes, comme le montre la pronominalisation possible de Quand-P par ce moment-là :

(11f) j'aime aussi beaucoup ce moment-là

(12a) je déteste ce moment-là

(13a) moi je préfère ce moment-là

Commutation impossible, ou sémantiquement difficilement acceptable, pour les propositions (14), (15) et (16) pour lesquels la pronominalisation de Quand-P est meilleure avec ce fait-là :

(14f) quand je vois des jeunes femmes chercher dans les poubelles, j'apprécie pas ça $\rightarrow$ ? j'apprécie pas ce moment-là

Mais : quand je vois des jeunes femmes chercher dans les poubelles, j'apprécie pas ce fait-là

(15a) Pour moi, un magistrat responsable, c'est quelqu'un qui, quand il est amené à commettre une faute dans l'exercice de ses fonctions, le reconnaît et en répond $\rightarrow$ ? Pour moi, un magistrat responsable, c'est quelqu'un qui, quand il est amené à commettre une faute dans l'exercice de ses fonctions, reconnaît ce moment-là

Mais : c'est quelqu'un qui quand il est amené à commettre une faute dans l'exercice de ses fonctions, reconnait ce fait-là

(16a) Nous n'avons pas la formule magique pour les battre, mais je peux vous assurer que, quand vous perdez deux fois contre une même équipe, vous n'aimez pas cela du tout $\rightarrow$ ? quand vous perdez deux fois contre une même équipe, vous n'aimez pas ce moment-là

Mais : quand vous perdez deux fois contre une même équipe vous n'aimez pas ce fait-là

Comme pour les structures en [Quand...ça/cela], la reprise anaphorique est situationnelle et non temporelle ainsi les propositions (11), (12) et (13) ne sont ni syntaxiquement (valence directe $v s$ structure corrélative), ni sémantiquement (interprétation temporelle $v s$ interprétation situationnelle) équivalentes aux propositions (14), (15) et (16). Il parait alors difficile d'envisager que les structures corrélatives soient dérivées des structures valencielles, mais la réponse possible à cette interrogation nous sera donnée après étude de la valence prépositionnelle. 


\section{Quand-P en position de valence prépositionnelle :}

En place de valence prépositionnelle on trouve les constructions suivantes avec les verbes de type de parler de, penser à... :

(17) Elle m'a parlé de quand vous étiez petits (M. Aymé, cité dans Le Petit Robert)

(18) Même que je les ai vus sniffer pendant le cours de maths dans un sac en plastoche. Moi y me suffisait de penser à quand je serais grande et que je serais devenue la collègue de mémé et qu'on ferait équipe toutes les deux... (Frantext : F. Seguin, L'arme à gauche, (1990 : 17-18))

Ici aussi un certain nombre de régularités sont observables dont la première : seules quelques prépositions semblent acceptées, dont $\grave{a}$ et $d e$ attestées.

Comme pour les précédentes structures, on remarque une contrainte temporelle, mais ici il ne s'agit pas de coréférence mais de concomitance, ce que l'on peut mettre en évidence, sur le modèle de A. Borillo (1988), par la commutation de quand par l'époque où, le moment où, le temps où... :

(17a) elle m'a parlé de quand/du temps-époque-moment où vous étiez petit

(18a) moi y me suffisait de penser à quand/au temps-moment-à l'époque où je serais grande

Pour ces propositions la Quand-P est difficilement mobile et son antéposition rend douteuse l'acceptabilité des énoncés, quand bien même on reprendrait pronominalement la Quand-P :

(17b) ? de quand vous étiez petit elle m'a parlé $\rightarrow$ ? de quand vous étiez petit elle m'en a parlé

$(18 \mathrm{~b})^{6}$ ? à quand je serais grande moi y me suffisait de penser $\rightarrow$ ? à quand je serais grande moi y me suffisait d'y penser

A moins d'une intonation vraiment particulière (17b) et (18b) sont difficilement acceptables. Cependant l'antéposition de Quand-P en valence prépositionnelle n'est pas interdite puisque l'on trouve en langue les propositions suivantes, où la Quand-P est reprise pronominalement dans la seconde partie de l'énoncé :

(19) Nicolas est somnambule, chuchoterait-on à la maison. On ne le dirait d'ailleurs pas devant lui : quand un enfant est gravement malade, on n'en parle pas devant lui. Dans quelle mesure était-ce grave, d'être somnambule? (Frantexte : E. Carrère, La classe de neige, $(1995: 102-103))^{7}$

(20) Il regardait devant lui, le corps très droit, la queue de cheval sur l'épaule, comme si depuis le départ il n'avait pas bougé. Quand Nicolas se redressa il s'en rendit certainement compte mais garda le silence. (Frantexte : E. Carrère, La classe de neige, (1995: 164-165))

(21) En 1996, quand la France à son tour signa un accord avec Moscou, ils eurent du mal à y croire (journal Le Monde, décembre 1999)

Que l'on peut trouver aussi avec les verbes suivants : s'y habituer, s'y intéresser, s'y fier, y songer, y rêver, s'en moquer, s'en méfier, s'en souvenir, en raffoler...

Comme pour les structure précédente il est possible pour (19), (20) et (21) d'intégrer l'adverbe certes :

(19a) quand un enfant est gravement malade, certes on en parle pas devant lui

(20a) quand Nicolas se redressa, certes il s'en rendit (certainement) compte

(21a) quand la France à son tour signa un accord avec Moscou, certes ils eurent du mal à y croire

Nous considèrerons ces propositions comme des structures corrélatives respectant le schème segmental [Quand-P + pronom anaphorique] et subissant la même contrainte temporelle de coréférence, que les structures corrélatives précédemment étudiées - contrainte qui ne s'applique pas sur les propositions (17) et (18) - comme le montrent les manipulations ci-dessous : 
(19c) ? quand un enfant était/sera gravement malade on n'en parle pas devant lui / ? quand un enfant est gravement malade on en parlait/parlera pas devant lui

(20c) ? quand Nicolas se redressait/redressera il s'en rendit certainement compte / ? quand Nicolas se redressa il s'en rendait/rendra certainement compte

(21d) ? quand la France à son tour signait/signera un accord avec Moscou, ils eurent du mal à y croire / ? quand la France à son tour signa un accord avec Moscou, ils avait/auront du mal à y croire

De plus, alors que les V1 et V2 de (17) et (18) sont dans un rapport de concomitance, pour (19), (20) et (21) il semble que ce soit plutôt un rapport de synchronisation qui soit mis en place, comme les manipulations (19d) à (21d) paraissent le montrer :

(19d) ? quand/le temps que-l'époque où un enfant est gravement malade on n'en parle pas devant lui

Mais : quand/tant qu'un enfant est gravement malade on n'en parle pas devant lui

(20d) ? quand/le temps que-l'époque où Nicolas se redressa il s'en rendit (certainement) compte

Mais : quand/tant que Nicolas se redressa il s'en rendit (certainement) compte

(21d) ? quand/le temps que-l'époque où la France à son tour signa un accord avec Moscou, ils eurent du mal à $\mathbf{y}$ croire

Mais : quand/tant que la France à son tour signa un accord avec Moscou, ils eurent du mal à y croire

Ici aussi, on retrouve le même système de reprise anaphorique que pour les Quand-P en valence directe, à savoir, lorsqu'elles sont construites en valence prépositionnelle, l'interprétation temporelle ne pose aucun problème - la Quand-P est pronominalisables par ce moment-là :

(17c) elle m'a parlé de ce moment-là

(18c) moi y me suffisait de penser à ce moment là

Par contre en structure corrélative, l'interprétation situationnelle prend le dessus sur l'interprétation temporelle, et il est meilleur de substituer Quand-P par ce fait-là :

(19d) quand un enfant est gravement malade on n'en parle pas devant lui $\rightarrow$ ? quand un enfant est gravement malade on ne parle pas devant lui de ce moment-là

Mais : quand un enfant est gravement malade on ne parle pas devant lui de ce fait-là

De plus pour (19d) la substitution du verbe etre par devenir rendrait la proposition acceptable puisque cette commutation rendrait à quand sa valeur temporelle. Cela va donc dans le sens d'une interprétation situationnelle pour (19).

(20d) quand Nicolas se redressa il s'en rendit (certainement) compte $\rightarrow$ ? quand Nicolas se redressa il se rendit (certainement) compte de ce moment-là

Mais : quand Nicolas se redressa il se rendit (certainement) compte de ce fait-là

(21d) quand la France à son tour signa un accord avec Moscou, ils eurent du mal à y croire $\rightarrow$ ? quand la France à son tour signa un accord avec Moscou, ils eurent du mal à croire à ce moment-là

Mais : quand la France à son tour signa un accord avec Moscou, ils eurent du mal à croire à ce fait-là

Pour les Quand-P en position de valence prépositionnelle on trouve donc deux structures concurrentes : une structure valencielle et une structure corrélative qui permet de construire la Quand-P avec un nombre important de verbes car «? je m'habitue à quand tu viens » parait plus difficilement acceptable que «quand tu viens, je m'y habitue », et qui permet surtout d'antéposer cette dernière. Cependant tous les verbes ne paraissent pas pouvoir accepter cette structure corrélative, comme les propositions ci-dessous en attestent :

(22) C'est souvent plus simple et/ou plus rapide d'utiliser les applis qu'on connait bien et de remettre à quand on a un peu de temps libre les expérimentations. (http://linuxfr.org/forums/41/14719.html)

(23) Alors je peux d'ores et déjà dire que mon plus lointain souvenir remonte à quand je vivais à Nîmes. (http://leeloolene.free.fr/index.php?2006/03/23/663-au-plus-profond-de-mes-souvenirs)

Car on imagine mal trouver (22a) et (23a) ci-dessous :

(22a) ? [...] de s'en/y remettre les expérimentations à quand on a un peu de temps libre

$\mathrm{Ou}: ?[\ldots] \mathbf{a ̀ ~ q u a n d ~ o n ~ a ~ u n ~ p e u ~ d e ~ t e m p s ~ l i b r e ~ d e ~} \mathbf{s}$ 'en/y remettre les expérimentations 
(23a) ? mon plus lointain souvenir en/y remonte à quand je vivais à Nîmes

$\mathrm{Ou}:$ ? à quand je vivais à Nîmes mon plus lointain souvenir en/y remonte

Ces derniers exemples apportent une réponse à la question posée précédemment : la structure corrélative ne parait pas être dérivée de la structure valencielle, mais constitue bien un schème structural à part entière, sinon il serait systématiquement possible de transformer une structure valencielle en corrélative, et inversement; de la même façon il est difficile de plaider pour une l'interprétation de Quand-P comme constituant thématisé. Nous avons vu qu'elle était topicalisée, ce qui induit une rupture avec ce qui précède, rupture non induite par l'opération de thématisation, qui implique le simple déplacement d'un constituant sujet ou objet.

\section{Conclusion}

Quand nous montre une fois de plus la richesse de ses emplois puisqu'il existe bien un tour corrélatif en [Quand-P + pronom anaphorique] et que ce schème permet de donner à Quand-P une plus grande autonomie énonciative (elle est topicalisée).

Cette structure corrélative présente une plus grande liberté structurale que la construction valencielle puisqu'elle permet l'antéposition ou la postposition systématique de la Quand-P. Par contre elle semble contrainte à la coréférence et au rapport de synchronisation entre V1 et V2, de même, la reprise pronominale est de l'ordre de l'anaphore associative, pointant non un moment, mais la situation ou l'état donné dans Quand-P.

Pour poursuivre cette étude, il conviendrait de regarder les types de relations discursives construites par les deux structures, valencielle et corrélative, et en quoi ces relations se distinguent ou se complètent. Il serait aussi intéressant de voir s'il existe une Quand-P corrélative concurrente des traditionnelles Quand$P$ circonstancielles. De même, il conviendrait de préciser les sémantismes et la nature exacte du morphème quand, qui, ici, ne saurait être considéré comme une simple « conjonction de subordination », si tant est que cette classe soit véritablement opérationnelle.

Effectivement quand invite ici à considérer des degrés d'intégration, de fait il serait peut-être probant de l'analyser sous la lumière de Lehmann (1988) qui postulait une échelle graduée d'intégration d'une construction verbale dans une autre («hierarchical downgrading»), échelle qui permet de dépasser heureusement la dichotomie traditionnelle entre hypotaxe et parataxe, partiellement inopérante pour la description des Quand-P, et dans laquelle la structure corrélative occupe une position centrale. Il convient donc d'ouvrir le sujet sur la question suivante: ne serait-ce pas la construction valencielle qui serait dérivée de la structure corrélative ? Haudry (1973) postulait déjà une réponse positive, pour le latin, à cette dernière interrogation.

\section{Références bibliographiques}

Allaire S., (1982), Le modèle syntaxique des systèmes corrélatifs, Thèse de doctorat, Univ. Rennes II.

Blanche Benveniste Cl., Deulofeu H.-J., Stéfanini J. et Eynde K. van den (1984), Pronom et syntaxe. L'approche pronominale et son application à la langue française, Paris : SELAF.

Benzitoun Christophe, (2007), Description morphosyntaxique du mot « quand » en français contemporain, Thèse de doctorat, Univ. Aix-Marseille I.

Borillo A., (1988), « Quelques remarques sur quand connecteur temporel », Langues Française, $n^{\circ} 77,71-91$ 
Charolles M., (1997), «L'encadrement du discours : Univers, Champ, Domaine et Aspect », Cahier de Recherche Linguistique, $n^{\circ} 6,1-73$

Combettes B., (1996), «L'intégration syntaxique de la subordonnée conjonctive topicalisée en français : approche historique », in C. Muller (éd.) Dépendance et intégration syntaxique, Niemeyer, Tübingen, 89-96.

Combettes Bernard, (1998), Les constructions détachées en français, Paris, Ophrys

Haudry J., (1973), «Parataxe, hypotaxe et corrélation dans la phrase latine », Bulletin de la société linguistique de Paris, $n^{\circ} 68,147-186$

Jeanjean C., (1985), « «Toi quand tu souris »: analyse sémantique et syntaxique d'une structure du français peu étudiée », Recherche sur le français parlé $N^{\circ} 6$, Aix en Provence, Presses Universitaires de Provence, 131-159

Le Draoulec A. et Bras M., (2006), "Quelques candidats au statut de "connecteurs temporels »", Cahier de grammaire « Spécial Anniversaire », 219-237

Lehmann C., (1988), "towards a typology of clause linkage", Typologocal studies in language, Vol18, Amsterdam/Philadelphia : John Benjamins publishing company.

Meillet A., (1921), Linguistique historique et linguistique générale, Paris : Klincksieck.

Muller Cl., (1983), «Les comparatives du français et la négation », Lingvisticae Investigationes 7: 2

Muller Cl., (1996a), La subordination en français, Paris : Armand Colin

Muller Cl., (1996b), Dépendance et intégration syntaxique. Subordination, coordination, connexion, Tübingen : Max Niemeyer Verlag.

${ }^{1}$ La terminologie utilisée ici est celle de l'Approche Pronominale (Claire Blanche Benveniste et al. (1987)) pour laquelle le verbe est une unité lexicale qui détermine et définit la forme de sa rection; cette dernière est alors posée comme une variable abstraite ayant pour représentation prototypique une proforme (opération de pronominalisation). Nous parlerons, pour ne pas multiplier les modèles, de valence directe et prépositionnelle.

${ }^{2}$ Terme initié par Cl. Muller (1983)

${ }^{3}$ Nous apportons une nuance pour l'exemple (5a) qui parait plus acceptable que (6a) : cette nuance vient peut-être de ce que le verbe surprendre peut se construire avec un sujet propositionnel, notamment une Que-P :

$\left(5 \mathrm{a}_{\mathrm{i}}\right)$ Que des êtres humains se comportent ainsi me surprend toujours

Cependant l'acceptabilité de $\left(5 \mathrm{a}_{\mathrm{i}}\right)$ va dans le sens de notre démonstration en ce que la $Q u e-P$ ne représente pas un moment mais une situation et nous verrons que ce qui distingue justement la Quand-P de (7) de la Quand-P de (5) c'est cette différence sémantique.

${ }^{4}$ B. Combettes (1996: 92): «[...] la proposition «principale», lorsqu'elle est précédée d'une subordonnée conjonctive topicalisée, offre les mêmes propriétés, les mêmes caractéristiques, qu'une indépendante : place et expression du sujet, utilisation d'adverbes modalisateurs ; ainsi que l'emploi de l'adverbe certes [avant la principale]. L'insertion de certes ne serait guère possible, dans un cas de thématisation entre le constituant initial [...] et le sujet, dans un schéma : Thème + Sujet + Verbe. »

${ }^{5}$ Notion établie par Cl. Blanche Benveniste et al. (1990:290) et qui correspond à «l'ensemble des réalisations valencielles d'un même verbe qui ont des parentés manifestes ». C. Benzitoun (2007 : 214), tout en reconnaissant l'ambiguïté de la notion de parentés manifestes confirme la transparence d'une telle notion et analyse sous son égide les propositions où la Quand-P est en position de valence objet direct avec la possibilité de construire la proposition de plusieurs manières (il inclut dans sa typologie les constructions des verbes interrogatifs que nous avons écartés ici). 
${ }^{6}$ L'acceptabilité plus grande de (18b) parait provenir de la présence de l'infinitif penser, mais si on conjugue ce verbe la proposition devient douteuse : ? « à quand je serais grande je pense ». (18b) ne nous parait donc pas aller à l'encontre de ce que nous avançons pour la valence prépositionnelle.

${ }^{7}$ En comparaison des propositions (17b) et (18b) l'acceptabilité des exemples (19), (20) et (21), pourrait s'expliquer par l'absence la préposition devant quand. Cependant une telle explication parait peu probante dans la mesure où, si l'on ajoute la préposition régime, le sémantisme des propositions change notablement :

(19) ? de quand un enfant est gravement malade on n'(en) parle pas devant lui

$\left(20_{\mathrm{i}}\right)$ ? de quand Nicolas se redressa il s'(en) rendit certainement compte

$\left(21_{\mathrm{i}}\right)$ ? à quand la France à son tour signa un accord avec Moscou ils eurent du mal à (y) croire

$\left(21_{\mathrm{i}}\right)$ est difficilement acceptable et pour $\left(19_{\mathrm{i}}\right)$ et $\left(20_{\mathrm{i}}\right)$ la présence de la préposition change le sens des énoncés par rapport aux propositions d'origine.

Il en va de même pour (17b) et (18b) dans la mesure où si on leur enlève les prépositions exigées par les verbes, le sémantisme est sensiblement différent, comme le montrent $\left(17 \mathrm{~b}_{\mathrm{i}}\right)$ et $\left(18 \mathrm{~b}_{\mathrm{i}}\right)$ ci-dessous :

$\left(17 b_{i}\right)$ ? quand vous étiez petit elle m'a parlé / ? quand vous étiez petit elle m'en a parlé

$\left(18 b_{i}\right)$ ? quand je serais grande moi y me suffisait de penser / ? quand je serais grande moi y me suffisait d'y penser

Pour $\left(17 \mathrm{~b}_{\mathrm{i}}\right)$ et $\left(18 \mathrm{~b}_{\mathrm{i}}\right)$ les Quand-P réfèrent à un instant donné et le sens des propositions est sensiblement différent de celui d'origine. Pour $\left(17 \mathrm{~b}_{\mathrm{i}}\right)$ on comprend qu'elle a parlé à son interlocuteur quand vous étiez petit (et non pas à un autre moment) et pour $\left(18 \mathrm{~b}_{\mathrm{i}}\right)$ on comprend qu'il suffit à je de penser dans la mesure où elle est grande, donc de sens très éloignés de ceux donnés en (17) et (18) :

(17) Elle m'a parlé de quand vous étiez petits (M. Aymé, cité dans Le Petit Robert)

(18) Même que je les ai vus sniffer pendant le cours de maths dans un sac en plastoche. Moi y me suffisait de penser à quand je serais grande et que je serais devenue la collègue de mémé et qu'on ferait équipe toutes les deux... (Frantext : F. Seguin, L'arme à gauche, (1990:17-18)) 